\title{
Transmission probability through small interacting systems: application to a series of quantum dots
}

\author{
Akira Oguri ${ }^{1}$ \\ Department of Material Science, Osaka City University, Sumiyoshi-ku, Osaka 558-8585, Japan
}

\begin{abstract}
We apply the theory for the transmission probability $\mathcal{T}(\epsilon)$ through small interacting systems, which was formulated based on the Kubo formalism in the previous study, to a series of quantum dots described by the $N$-impurity Anderson model. Specifically, we calculate $\mathcal{T}(\epsilon)$ for $N=2$ with the order $U^{2}$ self-energy and vertex corrections which satisfy the current conservation, and examine the two different parameter regions at $t\langle\Gamma$ and $t>\Gamma$. Here $t$ is the inter-dot transfer and $\Gamma$ is the level broadening caused by the coupling with the noninteracting leads.
\end{abstract}

Key words: transmission probability; mesoscopic system; Kubo formula; interacting electron systems; vertex correction

Recently, we have reformulated the conductance $g$ for small interacting systems connected to two noninteracting leads based on the Kubo formula [1]. Carrying out the analytic continuation of the vertex corrections following Éliashberg [2], we have obtained a Landauertype expression applicable to the interacting electrons,

$g=\frac{2 e^{2}}{h} \int_{-\infty}^{\infty} \mathrm{d} \epsilon\left(-\frac{\partial f}{\partial \epsilon}\right) \mathcal{T}(\epsilon)$,

where $f(\epsilon)$ is the Fermi function. The transmission probability $\mathcal{T}(\epsilon)$ is defined in terms of the vertex corrections or the three point correlation function [1], and depends on temperature $T$ in the interacting systems. Our derivation uses neither the precise form of the Hamiltonian nor the perturbation expansion, and thus the formulation can be applied to various systems such as quantum dots and atomic wires of nanometer size.

In this report we will apply the theory to the series of $N$ Anderson impurities, which can be regarded as a model for a network of quantum dots, and examine the parameter region that was not studied in the previous paper [1]. The system we consider consists of three regions; a small interacting region at the center $(C)$,

${ }_{1}$ E-mail: oguri@sci.osaka-cu.ac.jp and two noninteracting leads at left $(L)$ and right $(R)$. The total Hamiltonian is $\mathcal{H}_{\text {tot }}=\mathcal{H}^{0}+\mathcal{H}_{C}^{\text {int }}$, with $\mathcal{H}^{0}=$ $\mathcal{H}_{\mathrm{L}}+\mathcal{H}_{R}+\mathcal{H}_{C}^{0}+\mathcal{H}_{\text {mix }}$. Here, $\mathcal{H}_{L}$ and $\mathcal{H}_{R}$ are the Hamiltonian for the noninteracting leads. The central region and the connection to the leads are described by

$$
\begin{aligned}
\mathcal{H}_{C}^{0} & =-t \sum_{j=1}^{N-1}\left(c_{j+1 \sigma}^{\dagger} c_{j \sigma}+\text { H.c. }\right), \\
\mathcal{H}_{C}^{\text {int }} & =U \sum_{j=1}^{N}\left[n_{j \uparrow} n_{j \downarrow}-\left(n_{j \uparrow}+n_{j \downarrow}\right) / 2\right], \\
\mathcal{H}_{\text {mix }} & =-v \sum_{\sigma}\left(c_{1 \sigma}^{\dagger} c_{0 \sigma}+c_{N+1 \sigma}^{\dagger} c_{N \sigma}+\text { H.c. }\right),
\end{aligned}
$$

in the standard notation. Here the label $0(N+1)$ assigned to the site at the interface of the left (right) lead. The contributions of $\mathcal{H}_{\text {mix }}$ are described through the parameter $\Gamma=\pi \rho v^{2}$, where $\rho$ is the density of states of the isolated lead.

Specifically, in this report we consider the electronhole symmetric case and focus on the system of $N=$ 2. The conductance $g$ for the two-impurity Anderson model has been studied extensively. Particularly, the competition between the Kondo effect and the interdot magnetic exchange coupling occurring in this sys- 
tem has been investigated in detail based on the precise calculations by the numerical renormalization group method [3]. Nevertheless, the transmission probability $\mathcal{T}(\epsilon)$ has not been studied systematically, and as seen below it contains important information about the excitation spectrum.

To calculate $\mathcal{T}(\epsilon)$ from the expression which is written in terms of the Green's functions and vertex corrections [1], we take into account the order $U^{2}$ self-energy and vertex part illustrated in Fig. 1, taking $\mathcal{H}^{0}$ to be the unperturbed Hamiltonian. We note that the current conservation is satisfied in the calculations. In Fig. 2 the transmission probability at $T=0$ is plotted for $N=2$, where $U /(2 \pi t)$ is chosen to be (-) $0.0,\left({ }^{-} \circ-\right)$ 1.0 , and $(-\bullet-) 2.5$. The ratio of the mixing to the interdot transfer, $\Gamma / t$, is taken to be (a) 0.75 and (b) 1.25.

The low-energy behavior of $\mathcal{T}(\epsilon)$ is quit different depending on the value of $\Gamma / t$. As seen in Fig. 2 (a), for $\Gamma<t$ there are two resonant peaks at $\epsilon \simeq \pm t$, which for $U=0$ correspond to the bonding and anti-bonding states of the two dots. As $U$ increases, the height of these two low-energy peaks decreases, but the width of the peaks becomes sharp and the valley at $\epsilon=0$ becomes deep. Two additional broad peaks at high energy $\epsilon \simeq \pm U / 2$ appearing for $U /(2 \pi t) \gtrsim 1.0$ correspond to the upper and lower Hubbard levels of the atomic character. Fig. 2 (b) shows the results in the other parameter region $\Gamma>t$. For $U /(2 \pi t) \lesssim 1.0$, the transmission probability $\mathcal{T}(\epsilon)$ has only a single peak at low energies. The hight of this low-energy peak increases with $U$ until it reaches the unitary limit value. Then for $U /(2 \pi t) \gtrsim 1.0$ the hight of the peak decreases with increasing $U$, and eventually the single peak splits into two as seen in the results for $U /(2 \pi t)=2.5$.

The conductance can be obtained from eq. (1), and at $T=0$ it is determined by the value of $\mathcal{T}(\epsilon)$ at $\epsilon=0$. Thus for $\Gamma<t$ the conductance decreases monotonically with increasing $U$, while for $\Gamma>t$ it shows a maximum at the value of $U$ which corresponds to the unitary limit. At low temperatures, the $T$ dependence of the conductance is scaled by the energy determined by the width of the valley when $\mathcal{T}(\epsilon)$ has the two-peak structure at low energies. In the other case, for the single-peak structure, the energy scale is determined by the width of the central peak. We note that the temperature dependence of $\mathcal{T}(\epsilon)$ arises through that of the self-energy and vertex corrections, and at high temperatures the peak structure at low energies is smeared by the thermal fluctuations as demonstrated for $\Gamma / t=$ 0.75 in the previous paper [1].

The transmission probability $\mathcal{T}(\epsilon)$ we have formulated can also be written in terms of the three-point correlation function [1], and the Lehmann representation of it can be used for the nonperturbative approaches such as the numerical renormalization group and the quantum Monte Carlo methods.

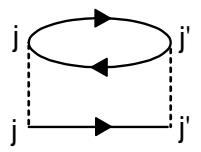

(a)

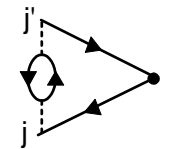

(b)

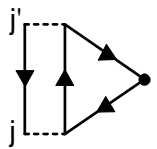

(c)

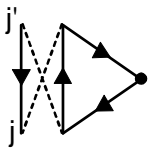

(d)
Fig. 1. The order $U^{2}$ corrections of (a) the self-energy and (b)-(d) the current vertex, where $1 \leq j, j^{\prime} \leq N$.
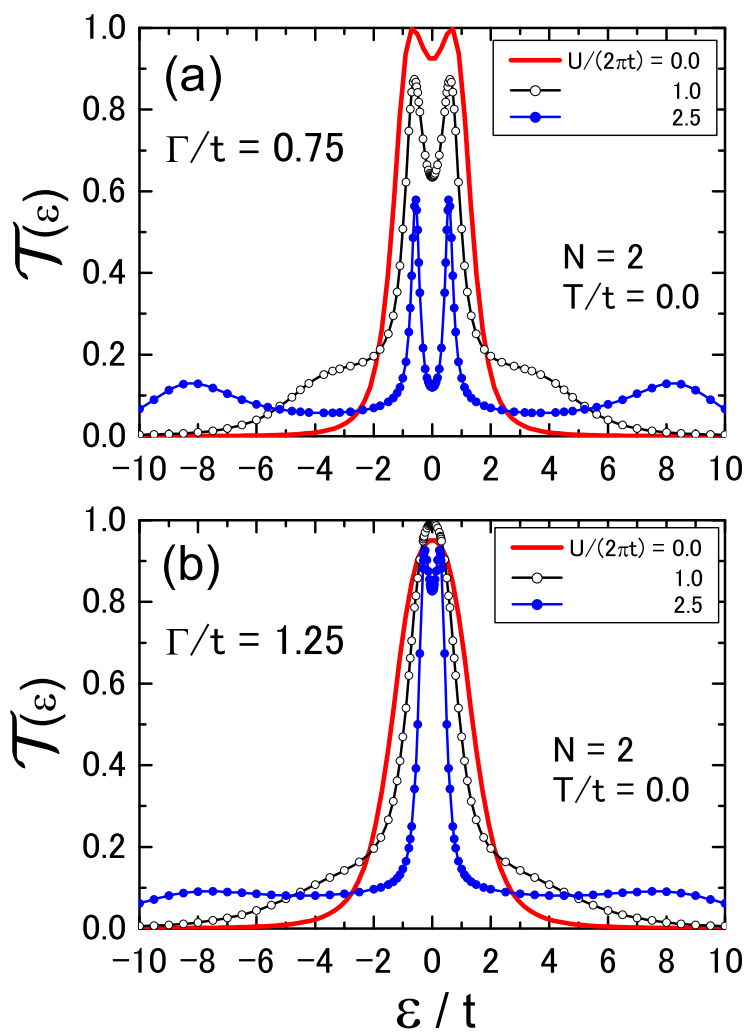

Fig. 2. Transmission probability for $N=2$ at $T=0$ for three values of $U /(2 \pi t) ;(-) 0.0,(-\circ-) 1.0$, and $(-\bullet-) 2.5$. The value of the ratio $\Gamma / t$ is taken to be (a) 0.75 and (b) 1.25 .

Acknowlidgements sions. Numerical computation was partly carried out at Yukawa Institute Computer Facility. This work is supported by the Grant-in-Aid for Scientific Research from the Ministry of Education, Science and Culture, Japan.

References

[1] A. Oguri, J. Phys. Soc. Jpn. 70 (2001) 2666. 
[2] G. M. Éliashberg, JETP 14 (1962) 886.

[3] W. Izumida and O. Sakai, Pys. Rev. B 62 (2000) 10260, and see also the references therein. 